\title{
The visual presence of determinable properties
}

Book or Report Section

Accepted Version

Stazicker, J. (2018) The visual presence of determinable properties. In: Dorsch, F. and Macpherson, F. (eds.)

Phenomenal Presence. Oxford University Press, Oxford. ISBN 9780199666416 doi: https://doi.org/10.1093/oso/9780199666416.003.0005 Available at https://centaur.reading.ac.uk/66597/

It is advisable to refer to the publisher's version if you intend to cite from the work. See Guidance on citing.

To link to this article DOI: http://dx.doi.org/10.1093/oso/9780199666416.003.0005

Publisher: Oxford University Press

All outputs in CentAUR are protected by Intellectual Property Rights law, including copyright law. Copyright and IPR is retained by the creators or other copyright holders. Terms and conditions for use of this material are defined in the End User Agreement.

\section{www.reading.ac.uk/centaur}

\section{CentAUR}

Central Archive at the University of Reading 
Reading's research outputs online 


\section{The Visual Presence of Determinable Properties}

James Stazicker

Forthcoming in Phenomenal Presence, eds. Dorsch, Macpherson \& Nida-Rümelin (OUP)

\section{The visual presence of a property}

Several essays in this volume exploit the idea that in visual experience, and in other forms of consciousness, something is present to consciousness, or phenomenally present to the experiencing subject. This is a venerable idea. Hume, for example, understood conscious experience in terms of the various items 'present to the mind'. ${ }^{2}$ However, it is not obvious how the idea should be understood ('present $t o^{\prime}$ is not a standard English modification of the adjective 'present') and there are grounds for worrying that there is no good way of making it precise. Here I explore a way of making precise the idea that properties of things, such as their shapes and colours, are present to us in visual experience. I argue that this important idea is coherent, well motivated and empirically plausible, provided that we reject two traditional assumptions: (i) that maximally determinate properties, rather than just determinable properties, are visually present; (ii) that we can tell through introspection exactly which properties are visually present to us.

For Hume, the qualitative character of visual experience consists in qualities of impressions which are present to your mind when you see. For example, where the impression that's present to you has a certain shape and colour, it seems to you that something with that shape and colour is before you, in such a way that the shape and colour figure essentially in the character of your experience: we have to mention those qualities if we're to characterise your experience faithfully. There is therefore a natural extension of Hume's claim that particular items are present to a subject, to the claim that qualities or properties are present to a subject. I'll understand the visual presence of a property $P$ to a subject $S$ as follows:

Presence It seems to $S$ that something before her has $P$, in such a way that a faithful description of the qualitative character of $S^{\prime}$ s visual experience must mention $P$.

This is a natural way to understand visual phenomenology: on the face of it, to describe the qualitative character of your visual experience faithfully, we have to state which properties things around you seem to you to have (Strawson 1979). The idea is also perennial in philosophy. One recent example: Susanna Siegel says that 'properties are presented in visual phenomenology (2010: 52); when she argues that certain properties are among those so presented, she takes her argument to establish what others have tried to establish by insisting on descriptions of the phenomenology which mention those properties

Passim, e.g. several instances at 1740/1978: 1.2.6.

'For the verb 'presented' in place of the adjective 'present', cf. e.g. Hume 1740/1978: 1.2.5. 
(2006: 485). I will limit my discussion to the idea that things' colours, shapes and sizes are visually present. Siegel, for example, argues that natural-kind properties are also among those presented in visual experience, but I want to avoid any controversy about that.

The idea that properties are visually present to us can seem absurd, if it is understood on the model of seeing particular objects. The visual presence of a property can then seem to require an impossible confrontation with a bare universal - the sort of perception imagined in uncharitable readings of philosophic cognition in the Phaedo and the Republic. But that is just the wrong model. Visual experience is experience of particulars, but it is also experience of general ways those particulars seem to be - ways which other things could be, such as red or round. Presence states a phenomenological restriction on things' seeming to be a general way. ${ }^{3}$

One might also hold that particular tropes or property-instances are visually present, in the sense that they figure essentially in the qualitative character of visual experience. That is left open by what follows. However, if the visual presence of a trope or instance of a general property $P$ is supposed to be independent of the visual presence of $P$, this introduces an alternative to the conception of phenomenology I'll explore. A particular instance of redness, say, is also a particular instance of many other properties. It might be the fourth output of the colour factory this morning, it might be hard to destroy, and so on. By treating its being red as what is visually present, we locate in visual phenomenology an explanation of why vision makes you sensitive, in belief and action, to that general property rather than the others. Merely treating the particular instance as visually present does not locate that explanation in visual phenomenology, if the visual presence of the particular instance is supposed to be independent of the visual presence of the general property.

Presence is compatible with various metaphysical theories about properties: having a general property might be instantiating a universal, being a member of a set, comprising a trope, or something else altogether. Presence is also consistent with various theories about the metaphysics of visual experience other than Hume's theory of impressions. For instance, Presence is consistent with the view that visual experience is a relation not to impressions and their properties, but to physical objects and their properties; on this view 'the qualitative character of the experience is constituted by the qualitative character of the scene perceived' (Campbell 2002: 114). Equally, the visual presence of a property may be explained in terms of the view that visual experience has an intentional content: $P$ is visually present because visual experience has an intentional content which attributes $P$ to your surroundings. ${ }^{4}$

\footnotetext{
Some philosophers argue that visual awareness of the general entails belief: to be visually aware of a general property is to see that something has that property, where seeing that $p$ is a species of knowing that $p$ (Stroud 2009). I think there is no such entailment, but what follows does not turn on this.

- This last approach is consistent with, but does not require, the view that the qualitative character of visual experience supervenes on its intentional content.
} 
The notion of the visual presence of a property is theoretically valuable, not just in characterising visual phenomenology itself, but also because it makes available an important starting-point for characterising the epistemic role of visual phenomenology: you can come to know that a property $P$ is instantiated before you, because $P$ is visually present to you. I assume that our claims about visual presence are subject to the following, related constraint: we should say that a property is visually present to a subject only where this contributes to a well motivated explanation of the judgements her visual experience enables her to make. As a result, the idea that properties are visually present faces a familiar challenge, in light of manifest limits on our capacity for visual discrimination.

For instance, suppose that under good viewing conditions you're presented with a series of many lines, First, Second, Third, ... Last. None of these lines seems to you to differ in length from either of its immediate neighbours: take any pair of neighbouring lines, and your visual experience does not dispose you to judge that they differ in length. Nonetheless, if the series is constructed appropriately, First and Last may visibly differ in length. If this is to be explained in terms of the visual presence of lengths, presumably the visually present length of First must differ from the visually present length of Last. But what about the other visually present lengths? On pain of incoherence, each of them must differ either from the visually present length of First or from the visually present length of Last. Therefore, the visually present lengths of at least two neighbouring lines must differ, even though these lines do not seem to differ in length. But how could the visual presence of lengths explain our judgements about length, if the visually present lengths of two lines may differ, without those lines seeming to differ in length?

To see the challenge here to the notion of visual presence, consider a standard approach to cases like First ... Last. Visual experiences of two objects may be 'phenomenally distinct', in that the experiences enable distinct patterns of judgement, even where the objects do not visibly differ (Goodman 1966: 272). For instance, your experiences of First and Second might be phenomenally distinct, even though First and Second don't visibly differ: perhaps the experience of First enables you to discriminate First from Last, while the experience of Second does not enable you to discriminate Second from Last. But what visually present lengths could contribute to a well motivated explanation of this pattern of judgement? On the face of it, if your experiences of First and Second are phenomenally distinct in that the visually present length of First differs from that of Second, the experiences should dispose you to judge that First and Second differ in length.

Parallel cases can be constructed for the visual presence of 2-D and 3-D size and shape, and for the visual presence of colour. So the challenge to the idea that properties are visually present generalises. Cases of this form can seem to show that the relationship between visual phenomenology and experienced properties must be more complex and less intimate than Presence allows. For instance, Christopher Peacocke has argued by appeal to 
such cases that visual experience of a length or colour should be characterised not in terms of the property currently experienced, but in terms of the experience's 'matching profile' the range of properties, most of them not currently visible, from which the experience does not enable you to discriminate a visible property (1989: 301ff). There are other challenges to the idea that properties are visually present, ${ }^{5}$ but the challenge I've stated is a central one, if the notion of visual presence is to earn its keep in terms of the psychological explanations we should expect from it. I'll show how we can meet the challenge, if we accept that both visual experience and introspection are in certain ways inexact: the properties visually present to us are determinable, and introspection does not reveal to us exactly which properties they are.

\section{Determination}

We can distinguish between a determinable property and its determinations (the properties which determine it). The property of being hexagonal determines the property of being shaped; being crimson and being scarlet each determine being red, being red determines being coloured, and being $6 \mathrm{~cm}$ long determines being between 5 and $10 \mathrm{~cm}$ long.

It's sometimes said that to have a property which determines a property $P$ is to have $P$ in a specific way. But to be red and square, say, is in a perfectly good sense to be red in a specific way. Being red and square does not determine being red. So this is not a sufficient condition on determination. Similarly, Stephen Yablo (1992) uses the following modal condition on determination, where ' $P$ ' and ' $Q$ ' express a determinable and its determination respectively:

$$
\square \forall x(Q x \rightarrow P x) \& \diamond \exists x(P x \& \sim Q x)
$$

Necessarily, everything crimson is red, but possibly - indeed actually - there are red things which are not crimson. But as Yablo acknowledges, this condition is not sufficient for determination as it has traditionally been understood (cf. Johnson 1921; Prior 1949). For example, Yablo's condition does not distinguish determinations from conjunctive properties like being red and square. Necessarily, everything red and square is red, and it's possible for things to be red without being red and square.

I'll work with a summary version of Eric Funkhouser's (2006) analysis of determination. We can think of a determinable property as having determination dimensions. These are variables along which a property is determined. For example, being coloured has the determination dimensions hue, saturation and brightness. Being coloured is a structurally simple case: to have any determination of being coloured is just to have values within some range for hue, saturation and brightness. Other properties are more complex, in that they're determined along a varying number of determination dimensions. Being (2-D)

¿See e.g. Travis 2004, to which I won't attempt to respond here. 
shaped has determination dimensions for number of sides, length of each side, and size of each interior angle; the number of dimensions for side-length and angle-size is a function of the number of sides. Still, being coloured and being shaped each fix at least the schematic determination dimensions I have described (Funkhouser 2006: 556). Length is among the simplest determinable properties, in that it has just one determination dimension: to have a determination of length is just to have a value within some range for length itself.

Property $P$ determines property $Q$ if and only if $P$ differs in nature from $Q$ only along the schematic determination dimensions of $Q$, such that the values along these variables consistent with instantiating $P$ are a proper subset of the values consistent with instantiating Q. Being scarlet differs in nature from being red only in that the range of hue, saturation and brightness consistent with being scarlet is a proper subset of the range consistent with being red. Being triangular differs in nature from being shaped only in that the number of sides and range of side-lengths and angle-sizes consistent with being triangular is a proper subset of those consistent with being shaped. Being between 9 and $11 \mathrm{~cm}$ long differs in nature from being between 8 and 12cm long, only in that the range of lengths consistent with being between 9 and $11 \mathrm{~cm}$ long is a proper subset of the range consistent with being between 8 and 12cm long. By contrast, being red and square differs in nature from being red in squareness, which is not a value for any schematic determination dimension of being red.

There is a traditional and persisting assumption about the properties visually present to us:

Determinacy If a determinable property $P$ is visually present in good viewing conditions, a determination of $P$ is visually present.

Determinacy says that where a colour or shape is visually present in good viewing conditions, a property corresponding to an absolute value, rather than merely a range, along the determination dimensions of that property is visually present. Where a colour is present, absolute values for hue, saturation and brightness are present. Where a shape is present, absolute values for number of sides, side-lengths and angle-sizes are present.

By contrast, if Determinacy is violated, what is visually present is just a property that's consistent with a range of absolute values along the determination dimensions in question. For instance, what is visually present might be the length between 9.995 and $10.005 \mathrm{~cm}$. This does not require that the upper and lower bounds of the range in question, or the unit of measurement, are also visually present - that we must mention these in a faithful description of your visual experience. Rather, what is visually present is a property which we, as interpreters, may describe in terms of its upper and lower bounds, measured in terms of certain units. To mark this, we might say that $P$ is visually present, and $P$ is in fact the

\footnotetext{
"This is only the gist of Funkhouser's analysis. As stated here, at least, the analysis is circular but informative. Unlike Johnson (1921), Funkhouser allows that distinct determinables may overlap in the way I exploit below. To that extent my use of 'determinable' differs from Johnson's.
} 
length between 9.995 and $10.005 \mathrm{~cm}$. But for ease of expression I won't always mark it that way.

Determinacy has a good claim to be the traditional view. For example:

'tis confest, that no object can appear to the senses; or in other words, that no impression can become present to the mind, without being determin'd in its degrees both of quantity and quality. The confusion, in which impressions are sometimes involv'd, proceeds only from their faintness and unsteadiness, not from any capacity in the mind to receive any impression, which in its real existence has no particular degree nor proportion.

Hume 1740/1978, 1.1.7

There are various ways to read this passage, but on one interpretation Hume here commits to Determinacy. In his view, sensory experience consists in impressions being present to the mind. Impressions are particular items, but the theory surrounding them generates Determinacy, a principle about the properties present to the mind. Like ordinary objects ordinarily conceived, impressions instantiate determinable properties only where they instantiate determinations of those determinables. Your impression is shaped only if it's shaped in a determinate way - square, for example. And for an impression to have a quality or property, that quality or property must be present to the mind. So if a determinable property is present to you, a determination of that property is also present to you. Restricting to the visual case, we get Determinacy.

Although few today would offer this argument for it, Determinacy remains tempting. It's tempting to think Determinacy is part of what's distinctive of visual experience, in contrast with thought or judgement. However, I'll show how rejecting Determinacy enables us to understand visual discrimination in a way which meets the challenge I sketched (§3). And I'll also argue that there are good grounds for rejecting Determinacy, independently of the challenge about discrimination $(\S 5)$.

\section{Determinables and discrimination}

There is a sense in which discrimination is the most basic visual capacity: in order to detect the various aspects of a visible scene, the visual system must respond differentially to them. Here we're concerned with a different sense of 'discrimination'. To discriminate $a$ from $b$ in this sense is to recognise that $a$ and $b$ are distinct, where this is a form of judgement or knowledge. Visually to discriminate $a$ from $b$ is to recognise that $a$ and $b$ are distinct on

I'll treat the absolute values corresponding to maximal determinates as absolute physical quantities. For Hume, the relevant absolute values are instead minima sensibilia, the most minute qualities of impressions one can experience (1740/1978: 1.2.1). If Determinacy is understood in terms of absolute physical quantities, then limited visual resolution is evidence against Determinacy (§5). If Determinacy is understood in terms of minima sensibilia, then varying resolution is evidence against Determinacy: there are episodes of visual presence in which the most determinate properties experienced are less determinate than those experienced at high resolution. I won't pursue that here. 
the basis of visual experience. We discriminate things with respect to their properties. For example, you might discriminate $a$ from $b$ by recognising that $a$ and $b$ differ in length.

One might think that we can discriminate things with respect to a determinable property wherever different determinations of that determinable are visually present. For example, one might think that you can discriminate $a$ from $b$ with respect to length, as long as the visually present lengths of $a$ and $b$ differ. But this leads quickly to paradox in the face of cases like First ... Last. Here's the case again. You're presented with a series of many lines: First, Second ... Last. None of them seems to differ in length from either of its immediate neighbours, but First and Last visibly differ in length. So the visually present length of First differs from the visually present length of Last, and on pain of incoherence, each of the other visually present lengths must differ either from that of First or from that of Last. Therefore, the visually present lengths of at least two neighbouring lines must differ. Yet you cannot discriminate these neighbouring lines with respect to their lengths.

A more promising approach becomes available if we reject Determinacy, and suppose that visual discrimination is to be explained in terms of the visual presence of determinable properties. Determinables have the following useful feature:

Incompatible If $P$ and $Q$ each determine some property, and no property determines both $P$ and $Q$, then nothing could have both $P$ and $Q$ simultaneously, in the same part and in the same respect.

In a property space, this is reflected by the fact that $P$ and $Q$ correspond to non-overlapping regions. For example, nothing could have a part which was simultaneously both between 9.995 and $10.005 \mathrm{~cm}$ long and between 10.015 and $10.025 \mathrm{~cm}$ long, in the same respect; nothing could be coloured in such a way that it was both turquoise and scarlet all over at the same time, and in the same respect. Where $P$ and $Q$ meet this condition I'll say that $P$ and $Q$ are incompatible. Where $P$ and $Q$ each determine some property but fail Incompatible, I'll say that they're compatible. Compatible properties share some determinations (they overlap in property space), so it's possible to have them simultaneously, in the same part and in the same respect.

I propose that we discriminate according to the following rule:

Discrimination Judge $a$ different from $b$ with respect to $D$, where $a$ and $b$ have visually present and incompatible determinations of $D$.

For example, suppose $a$ has the determinable length between 9.995 and $10.005 \mathrm{~cm}, b$ has the determinable length between 10.015 and $10.025 \mathrm{~cm}$, and these lengths are visually present. This entails that $a$ and $b$ differ in length. Accordingly, you could discriminate $a$ from $b$ with respect to length by following Discrimination. By contrast, if the visually present lengths of $a$ and $b$ are compatible determinables, $a$ and $b$ might have the same exact length. Accordingly, you cannot discriminate $a$ from $b$ with respect to length by following Discrimination. For 
example, suppose $a$ has the determinable length between 9.995 and $10.005 \mathrm{~cm}, b$ has the determinable length between 10.002 and $10.012 \mathrm{~cm}$, and these lengths are visually present. $a$ and $b$ might have the same exact length, and accordingly, you cannot discriminate $a$ from $b$ with respect to length by following Discrimination.

We don't usually think in terms of Discrimination (any more than we usually think in terms of a rule about different visually present properties). Rather, I propose that which properties are visually present is one of the factors influencing how things look. In particular, where $P$ and $Q$ are incompatible and visually present, the objects which have $P$ and $Q$ typically seem to differ with respect to a determinable of $P$ and $Q$. Judging on this basis that the objects differ with respect to that determinable, you judge in accordance with Discrimination.

Figure 1

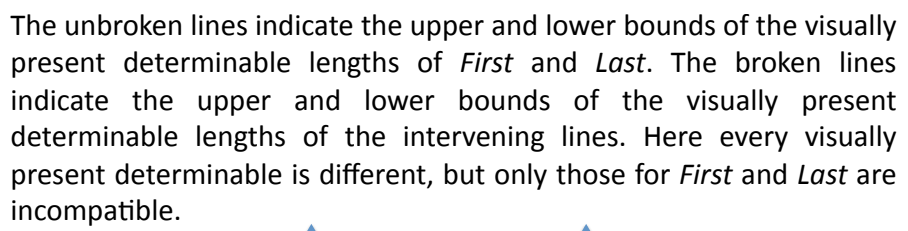
incompatible.

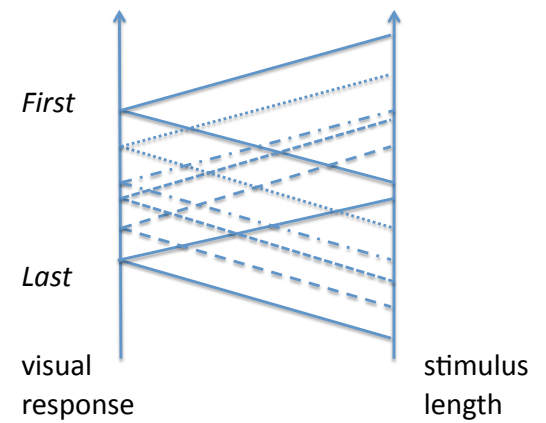

So here is the first step towards meeting our challenge. The visually present length of at least one line between First and Last must differ either from the visually present length of First or from that of Last, but lines do not in general seem to differ where their visually present lengths are merely different, rather than incompatible. First and Last, by contrast, are discriminable because their visually present lengths are incompatible. This may be the case while no visually present length in the series is incompatible with either of its immediate neighbours; what's required is just that some immediate neighbours differ. In fact, the visually present length of every line in the series could be different from every other (Figure

\footnotetext{
- This is not to say that following Discrimination is always necessary for discriminating. There may be cases in which one can discriminate things probabilistically on the basis of their visual present compatible properties (see Stazicker 2011, Ch.5). In the example just given, $b^{\prime}$ s being longer than $a$ is consistent with most determinations of the two visually present lengths, so to the extent to which background conditions have parallel effects on your experiences of $a$ and $b, b$ is probably longer than a. Since one cannot reliably assume that background conditions have entirely parallel effects on such experiences, this probabilistic method of discrimination will always be unreliable where there is more than a certain degree of overlap between visually present lengths. That degree of overlap, rather than compatibility, will then mark the point at which one cannot discriminate. Following Discrimination may not always be sufficient for discriminating either - see fn.17.
} 
1). In principle, we could meet parallel challenges about the visual discrimination of properties other than length in the same way, since compatibility and incompatibility are well defined for all determinable properties. The discrimination of length is distinctive only in that length is a very simple determinable.

I'll argue that this response to cases like First ... Last is uniquely well motivated, when compared with alternative responses which seek to defend the notion of visual presence while insisting on Determinacy. I'll return to the alternatives in §4, but I want now to set aside a popular alternative response (Jackson and Pinkerton 1973; Graff 2001). According to this response, experience is shifty: experience of at least one line in the series changes, depending on which of its neighbours you're comparing it with. For instance, your experience of the length of Second might be identical with your experience of the length of First when those two are compared, but differ so that it's identical with your experience of the length of Third when Second and Third are compared. That explains why First and Second don't visibly differ, and it explains why Second and Third don't visibly differ. And it doesn't require, incoherently, that the experience of Second be simultaneously both the same as that of First and different from it.

Whatever its other advantages, this approach does not provide a good alternative defence of the notion of visual presence. For either the hypothesised shifts in experience entail widespread illusion, or we must reject Determinacy, making the hypothesis of shiftiness obsolete.

Suppose the visually present lengths of First, Second and Third are maximally determinate. For instance, the visually present length of First is (exactly) $9 \mathrm{~cm}$, and that of Third is (exactly) $10 \mathrm{~cm}$; the visually present length of Second shifts from $9 \mathrm{~cm}$ to $10 \mathrm{~cm}$ depending on which neighbour you compare it with. Then if the length of Second in fact remains constant, at least one experience here must be illusory: under good conditions visual experience does not present things as they are.

Pending clear evidence to the contrary, we should assume that visual experience normally presents things as they are. One set of reasons for this lies in substantive theories of what it is for a property to be visually present. If the visual presence of $P$ consists in the subject's being related to $P$ as $P$ is instantiated by the environment, then where $P$ is visually present the environment invariably instantiates $P$. And in some versions of the intentional theory, for an experience to attribute $P$ is (in part) for the experience reliably to indicate things' having $P$ (Fodor 1990; Dretske 1995). If visually present properties are then held to be properties which visual experience attributes, $P$ is visually present only where this visual response is reliably correlated with the environment's instantiating $P$. In addition, independently of these theories it's a basic principle of interpretation that, when we ascribe semantic values to states of a system, we do so in such a way that they come out by-and- 
large veridical. Things can go wrong but veridical appearances should be the norm, pending good reason to suppose that appearances are illusory.

We can respect this if we reject Determinacy, and suppose that the lengths visually present to us are determinable. Then visually present lengths may shift while experience nonetheless presents things as they are. For instance, if Second is exactly $10 \mathrm{~cm}$ long, you might experience it now as $8-11 \mathrm{~cm}$ long, then as $9-12 \mathrm{~cm}$ long, all the while experiencing things as they are. But once we deny Determinacy, the hypothesis of shifty experiences is obsolete. A coherent account of visual discrimination in cases like First ... Last is available with or without that hypothesis: we discriminate in accordance with Discrimination.

The response I've proposed to cases like First ... Last is just one version of an idea which need not be understood in terms of visual presence: the idea that we discriminate on the basis of visual experiences which fail to specify the exact character of our environment. Where our aim is not to defend the notion of visual presence, the crucial notion of overlap in a property space need not be spelled out in terms of the visual presence of determinable properties. For example, the account I've proposed owes a great deal to Benj Hellie's solution to a puzzle about colour experience: you're presented with three colour samples, $a$, $b$ and $c$, such that $a$ does not visibly differ in colour from $b$, nor $b$ from $c$, but $a$ visibly differs in colour from $c$. Hellie's solution turns on the claim that colour experience is unspecific: visual experience specifies that the colour before you lies within a certain region of colour space, but fails to specify any exact point in colour space at which the colour lies. Hellie claims that the regions specified for indiscriminable colours overlap, while those for discriminable colours do not overlap. Since overlap is not a transitive relation, region $A$ may overlap with $B$, and $B$ with $C$, while $A$ does not overlap with $C$. Hence the colour of $a$ may be indiscriminable from that of $b$, and the colour of $b$ may be indiscriminable from that of $c$, while the colour of $a$ is discriminable from that of $c$ (Hellie 2005: 485-6).

Hellie does not defend the idea that determinable colours are visually present - nor, for that matter, the idea that any colours are visually present. He argues that the properties represented in colour experiences are maximally determinate colours, and yet they are not represented maximally determinately' (489). This does not require that the maximally determinate colour is visually present - that we must mention it in a faithful description of the phenomenology. From the subject's point of view, it's obscure exactly which colour this is. But neither does this approach require that a determinable corresponding to the relevant region of colour space is visually present. For instance, one way to represent a maximally determinate colour as lying within a certain region of colour space is to mention the several maximally determinate colours which it could be, consistent with this way of representing it. No mention is required of a determinable colour which the object before one seems to have.

Still, I am arguing that we can defend the idea that properties are visual present using an account which draws, like Hellie's, on the notion of overlap in property space. In 
terms of visually present determinable colours, the solution to the puzzle about colour experience would run as follows: the visually present colours of $a, b$ and $c$ may all be different, while only the visually present colours of $a$ and $c$ are incompatible; by following Discrimination, you can discriminate only $a$ and $c$ with respect to colour.

However, two disputes with Hellie will emerge in what follows. An account of the general type we both propose is plausible only to the extent to which it's plausible that visual experience is unspecific. I'll argue that this is plausible with respect to the experience of spatial properties (§5) but should be more controversial than Hellie allows with respect to colour experience (\$6). Furthermore, an account of this general type is plausible only if introspection fails to specify accurately the exact character of our visual experiences, just as visual experience fails to specify accurately the exact character of our environment $(\S 4)$.

\section{Inexact introspection}

To see the implications of cases like First ... Last for our powers of introspection, consider an objection to my account of these cases that's due to Christopher Peacocke (1989). Peacocke argues that it's incoherent to characterise visual experience in terms of precise experienced properties, even if these properties are unspecific or determinable. (A determinable is precise if it has exact bounds - if it occupies a definite region of property space. For instance, the determinable length between exactly 9.995 and exactly $10.005 \mathrm{~cm}$ is precise. My account of First ... Last is consistent with the claim that precise determinable properties are visually present, so my account falls within Peacocke's target.)

Peacocke's example is visual experience of the direction in which something lies from you:

In fact the idea of the precise direction experienced is incoherent in a familiar way if it is supposed to conform to the following principle: that the members of a pair of such directions are the same if and only if they seem to be the same. ... For any dimension along which matching (nondiscriminable difference) is nontransitive, contradiction results if we suppose there are shades or precise directions experienced conforming to that principle. If $a$ matches $b, b$ matches $c$ but $a$ does not match $c$, there is no precise direction experienced coherently assignable to $a$, since it must both be the same as that of $c$ (by virtue of being the same as that of $b$, which both $a$ and $c$ match), and different from it (by virtue of $a$ 's not matching $c$ ).

Peacocke 1989: 301

Peacocke appeals to a principle that 'directions experienced ... are the same if and only if they seem to be the same'. On this basis he infers that:

(1) the direction experienced for $a$ is the same as that for $b$;

(2) the direction experienced for $b$ is the same as that for $c$;

(3) the direction experienced for $a$ is different from that for $c$. 
But by the transitivity of sameness, (1) and (2) entail:

(4) the direction experienced for $a$ is the same as that for $c$.

Since (3) and (4) are inconsistent, Peacocke rejects the idea that there are precise directions experienced, arguing that the relationship between visual experience and currently visible properties must be more complex and less intimate than Presence allows (1989: 302ff).

This argument raises an issue about the transitivity of sameness, which I'll mention briefly if only to set it aside. The relevant notion of sameness is introduced by reference to pairs of indiscriminable directions. So the argument legitimately treats sameness as transitive, only if there is a sense of 'seem to be the same' which applies to directions where they are visually indiscriminable and which expresses a transitive relation.

It's not clear that there is such a sense. Visually indiscriminable directions need not seem to be exactly the same. For as Peacocke recognises (1989: 301) vision is not arbitrarily powerful and so does not inform us of things' exact directions. But we can legitimately describe things as the same without implying that they're exactly the same. For example, you might tell me that two people are the same height without suggesting that they're exactly the same height. You should be understood as allowing that the two heights may differ, as long as the difference is within a reasonable margin. So the thought might be that directions seem to be the same, where their appearance is inconsistent with their differing by more than a certain (perhaps contextually defined) angle $M$. However, it's not clear why we should think that being the same in this sense is transitive. Suppose the visual appearance of $a$ and $b$ is inconsistent with their differing by $>M$, and the visual appearance of $b$ and $c$ is inconsistent with their differing by $>M$. Why should the visual appearance of $a$ and $c$ not be consistent with their differing by $>M$ ? Compare: You believe that Ahmed is the same height as Boris, to within an inch, and that Boris is the same height as Cindy, to within an inch. You may consistently hold that Ahmed is not the same height as Cindy, to within an inch.

The semantics of 'seem to be the same', 'look the same' and their ilk are not straightforward, and I propose no account of them here. In general, it's controversial whether there is a sense of these expressions which (a) applies to properties where they are indiscriminable and (b) is transitive. So it is not clear whether the last stage of Peacocke's argument goes through. However, for current purposes we need not worry about this last

\footnotetext{
- Jackson and Pinkerton (1973) assume the conjunction of (a) and (b). Wright (1975) rebuts it. Mills (2002) exploits the conjunction of (a) and (b) to defend a claim that's related to my conclusion in this section, though distinct from it: we're introspectively fallible about whether things look the same. Graff (2001) assumes (a) and defends (b). Graff criticises arguments against the transitivity of 'look the same' which appeal to 'our putative inability to perceptually detect [i.e. perceive] very slight changes'. I will appeal instead to insensitivity to slight changes, to argue that the monadic spatial properties visually present to us are determinable (\$5). I'll then exploit that conclusion, in turn, to motivate the account of visual discrimination proposed in $\$ 3$, according to which visual indiscriminability is intransitive.
} 
stage of the argument, since (1) and (2) above are enough to challenge the idea that properties are visually present. According to Peacocke, (1) the directions experienced for $a$ and $b$ are the same, and (2) the directions experienced for $b$ and $c$ are the same. By contrast, any plausible account of visual presence requires that the visually present directions of $a$ and $b$ differ (if they didn't, $b$ 's direction would, like $a^{\prime}$ s, be discriminable from $c^{\prime}$ s), and that the visually present directions of $b$ and $c$ differ (if they didn't, $b^{\prime}$ s direction would, like $c^{\prime}$ s, be discriminable from $a^{\prime}$ s). For example, on the account I proposed we should understand Peacocke's case as follows:

(1') The visually present directions of $a$ and $b$ are different but compatible.

$\left(2^{\prime}\right) \quad$ The visually present directions of $b$ and $c$ are different but compatible.

(3') The visually present directions of $a$ and $c$ are both different and incompatible.

Peacocke infers (1) and (2) by appeal to his principle that 'directions experienced ... are the same if and only if they seem to be the same'. For our purposes, only half the biconditional is crucial: directions experienced are the same if they seem to be the same. Peacocke uses the expression 'directions experienced' to refer to 'the contents of the experiences' (301) - in our terms, to the directions which are visually present. So Peacocke's principle does not just concern our capacity to detect whether objects differ in their visible features; it concerns our capacity to detect whether experiences differ in their contents. In that sense, Peacocke's principle concerns our capacity for introspection. The general principle is that, with respect to their contents, simultaneous experiences are the same if they seem introspectively to be the same. In our terms:

Access Simultaneously visually present properties are the same, if they seem introspectively to be the same.

Now if Peacocke's principle is to license (1) and (2), the principle must be more complex than it first appears, because it is not trivial that Peacocke's case is one in which the contents of two experiences seem to be the same. In the first instance, the case is one in which two objects of experience do not visibly differ in direction. If it's to follow that two directions experienced seem to be the same, we need to add something along the following lines: two directions experienced seem to be the same, if the objects of experience do not visibly differ in direction. In our terms:

Transparency Simultaneously visually present properties seem introspectively to be the same, if the objects of experience do not visibly differ with respect to those properties.

Together, Access and Transparency entail that visually present properties are the same, wherever things do not visibly differ with respect to those properties. For instance, the visually present directions of $a$ and $b$ are the same, and so are the visually present lengths of 
neighbouring lines in First ... Last. A coherent account of visual presence requires that these visually present properties differ. So a coherent account of visual presence must deny either Access or Transparency.

Transparency is an extension of G.E. Moore's famous comment that introspecting the experience of blue reveals only the blue (1903): Transparency says that introspection reveals a difference between visually present properties only where it reveals an apparent difference in the visible world. One could accept Moore's claim without accepting Transparency, since Moore's claim concerns the first-order property of being blue, while Transparency concerns the second-order property of differing in a first-order property. And there is reason to doubt that Transparency is true without qualification. Take a case in which $a$ and $b$ do not visibly differ in length, and you have a good view of $a^{\prime}$ s length but a poor view of $b^{\prime}$ s length ( $b$ might be far away, badly lit, or at an awkward angle). This difference between your experiences of the lengths is introspectable, and on the account I'm proposing it is plausible that the difference consists partly in different lengths' being visually present: the length of $a$ that's visually present is more determinate than the length of $b$ that's visually present.

However, this kind of difference between visually present lengths is not the kind of difference at work in First ... Last. We can distinguish between two ways in which properties may differ, corresponding to dilation and translation in a property space. The properties 4 to 8 metres long and 5 to 7 metres long are dilations of one another; the latter is more determinate than the former. By contrast, the properties 4 to 8 metres long and 6 to 10 metres long are translations of one another. So are the properties 4 to 8 metres long and 10 to 14 metres long, and the maximally determinate properties 4 metres long and 6 metres long. This distinction is well defined, in terms of the operations of translation and dilation, not just for the one-dimensional property space of length, but for $n$-dimensional property spaces.

The kind of difference between visually present lengths that's at work in the uniformly good conditions of First ... Last is translation rather than dilation. Incompatible lengths are translations of one another. So the visually present lengths of First and Last differ by translation, and the visually present lengths of at least two neighbouring lines in the series must differ by translation, even though those lines do not visibly differ in length. As a result, a principle that's weaker than Transparency will suffice for our purposes:

Transparency* Simultaneously visually present properties do not seem introspectively to differ by translation, if the objects of experience do not visibly differ with respect to those properties.

Figure 2

$$
\text { ////////////////////////////// }
$$


Together, Access and Transparency* entail that visually present lengths of neighbouring lines in First ... Last do not differ by translation. ${ }^{10}$ Since the visually present lengths of some neighbouring lines do differ by translation, a coherent account of visual presence must deny either Access or Transparency*.

There is a compelling introspective case for Transparency*. When reflection on experience reveals that two translated lengths, say, are visually present, reflection on experience also reveals the appearance that the objects of experience differ with respect to length. For instance, some of the lines in Figure 2 appear to differ in length, while others do not; it is introspectable that the apparent lengths of the lines are translations of one another only in cases where the lines appear to differ in length. In the remaining cases, just as there is no apparent difference in length, so there is no introspectable translation between the lengths which are apparent. I think broader reflection on experience reveals that what applies to Figure 2 also applies elsewhere, and with respect to other visible properties.

Transparency* is a very minimal claim. It allows that some aspect of your experiences of length may vary in a way that's introspectable, while no difference in objects' lengths is apparent. The allowed introspectable differences are not limited to dilations among visually present lengths. For instance, if one object is closer to you than another, your experience of each object's length may differ, while no difference in objects' lengths is apparent, in that one of the objects occupies more of your field of view than the other. We could take this to be a difference in some perspectival aspect of what the experiences present: experience presents one of the objects as closer, or as occupying a larger portion of what's visible from here. Or we could take this to be a purely phenomenal difference between the experiences, over and above any difference in what the experiences present (cf. Peacocke 1983). Either way, this is consistent with Transparency*. There is no introspectable translation between visually present lengths, unaccompanied by an apparent difference in length.

If we accept Transparency*, and we want to explain visual discrimination in terms of the visual presence of properties, we must reject Access: the properties visually present to us sometimes differ by translation, even though the objects of experience do not visibly differ with respect to these properties; this difference between visual experiences is not introspectable. Just as visual presence does not lay bare the properties of our environment in all their determinate detail, so that every difference between them is apparent, similarly visual presence does not lay bare our experiences in all their determinate detail, so that every difference between them is apparent. The subject of experience does not possess full knowledge of which properties are visually present to her - of which properties must be mentioned in a faithful characterisation of her own visual experience."

\footnotetext{
10 Assuming, with Peacocke, that things which don't seem to differ seem to be the same. That assumption could be dropped, and Access reformulated in terms of not differing.

" Hellie denies 'higher order indiscriminability' of this kind, in effect by denying Transparency* (2005: 497, fn.23). Where colours are indiscriminable though experiences of those colours differ by
} 
The analogy between vision and introspection should not be pressed too far. Introspection, as I am understanding it, is any distinctively first-personal awareness of one's own experiences. So it is artificial to treat introspection as a form of inner sense, analogous to vision in all the relevant respects. For example one model would take introspection, like vision, to be an awareness of determinable properties. (In the case of introspection, the objects of awareness would be determinable experiences, corresponding to regions of the space of possible experiences; points in the space, or maximally determinate experiences, would include the visual presence of determinable properties of the environment.) Or more generally, we might suggest that introspection is unspecific about which experiences you have - i.e. that introspection presents an experience as being of a certain unspecific type, without specifying which exact type of experience it is. I won't pursue any such account of the contents of introspection here. I propose only that introspection is inexact in the following sense: even in good conditions such as those of First ... Last, introspection does not specify accurately exactly which properties are visually present to you. For if in such good conditions introspection specified accurately exactly which properties are visually present to you, this would enable you to detect translations among the visually present properties of neighbouring stimuli in cases like First ... Last.

Once the choice between Access and visual presence is articulated, it is unclear why one would insist on Access. In $\S 1$, I said that the notion of visual presence makes available a simple account of the epistemic role of visual experience: you can come to know that a property $P$ is instantiated before you, because $P$ is visually present. If we think of this knowledge as consisting in a capacity to name or describe $P$ precisely, then perhaps it's implausible that introspection should be limited in the way I'm proposing. Someone rational who had that capacity could presumably also tell that $P$, rather than any different property, was visually present to her. But we need not understand knowledge of visible properties in that way. When you know that a certain spatial property is instantiated before you, because that property is visually present to you, this knowledge consists in a capacity to make certain judgements of distinctness, and certain judgements about an object's causal role - for example, judgements about the spaces in which the object will fit.

translation, Hellie claims that 'there is a clear introspectable difference between the phenomenal characters (with respect to colour) under which the colours ... are presented'; ... 'the regions [of colour space] they are presented as in differ'. In support of this, Hellie appeals to his view that there is 'phenomenal noise' in visual experience, a 'flickering or crepitation' which obscures exact colours (see fn.19 below). He claims that where two experiences specify different (but overlapping) regions of colour space, there is an introspectable difference between how the two experiences flicker. Let's accept, for the sake of argument, that there is flickering of this kind in colour experience, and that differences in it are introspectable wherever experience specifies different regions of colour space. Then experiential differences which occlude colour are introspectable. But it doesn't follow, against Transparency*, that you can introspect translations between experienced colours themselves, in cases where you cannot discriminate objects with respect to colour. If flickering is a form of noise, two experiences might flicker in different ways, yet specify the same region of colour space. So introspectable flickering is no guarantee of an introspectable translation between experienced colours. 
To defend Access one might appeal to broadly Fregean constraints on mental content: one might insist that differences in visual experience are cognitively significant, and so genuine, only for someone able to recognise those differences. However, differences in what is visually present may be cognitively significant without being introspectable. For instance on the account I have proposed, where two different but compatible determinable properties are visually present to you, this difference is not introspectable. But each experience puts you in a position to make a different set of discriminations, since different but compatible properties are each incompatible with a different set of further properties.

I have argued that any plausible account of visual presence must reject Access. Now rejecting Access makes room for a coherent account of visual presence, whether or not we also reject Determinacy. For example, one could maintain Determinacy and coherently understand the case of First ... Last as follows. Each visually present length in the series is maximally determinate, and the visually present lengths of some neighbouring lines differ by translation, but things visually appear to differ in length only where their visually present lengths differ by more than a margin for error $M$; the visually present lengths of neighbouring lines differ by $<M$, while the visually present lengths of First and Last differ by $>M .{ }^{2}$ By Transparency*, this interpretation requires that you cannot introspect differences $<M$ in the lengths visually present to you. But there is nothing incoherent about that, given the failure of Access.

However, this sort of interpretation is not well motivated (§5). Given the failure of Access, nothing about the introspectable character or functional role of visual experience motivates the claim that maximally determinate lengths are visually present. By contrast, the functional role of visual experience does motivate the claim that determinable lengths are visually present. So given the failure of Access, a solution which denies Determinacy is well motivated, and a solution which insists on Determinacy is not.

\section{Determinable properties and spatial resolution}

As I said (§3), pending evidence to the contrary we should expect that normally, where a property $P$ is visually present, the environment instantiates $P$. Where this correlation holds, it will be supported by the fact that $P$ makes a difference to your visual responses. I'll say that a visual response $R$ is sensitive to a property $P$ where:

' Cf. Williamson's (2013a) model, which treats visual knowledge as leaving a margin for error in maximally specific visual appearances. But note that the interpretation of First ... Last requires an account of things' visually appearing to differ, not just of things' knowably differing. So this interpretation of First ... Last requires that appearances of difference in length are more conservative than appearances of individual lengths: vision refrains from presenting you with differences in length where they fall within the margin for error; vision does not refrain from presenting you with maximally determinate monadic lengths even though they may be in error by the same margin. Perhaps that asymmetry is objectionable, but I won't pursue the point here. 
Sensitivity In every background condition such that $R$ occurs in the normal way, if $P$ had not been instantiated, $R$ would not have occurred.

On a standard semantics for the counterfactual (Lewis 1973), and where the instantiation of $P$ is a contingent matter, Sensitivity is true iff:

For every possible world $w$ at which background conditions are such that $R$ occurs in the normal way, there is at least one world $x$ at which $P$ is not instantiated and $R$ does not occur, such that $x$ is closer to $w$ than every world at which $P$ is not instantiated and $R$ does occur.

Because of the limited spatial resolution of vision, our visual responses are sensitive only to determinable shapes and sizes. The spatial resolution of a response is given by the maximum spatial frequency in a stimulus at which the response is sensitive to variations in the stimulus (De Valois and De Valois 1988). Intuitively, we can think of the spatial frequency of a stimulus as its rate of variation across space. For example, where a stimulus has a higher spatial frequency, it typically has a more detailed pattern. In Figure 3, spatial frequency increases from left to right, in that the rate of variation from light to dark and back increases from left to right. Assuming its spatial resolution is high enough, your visual system is sensitive to variations on the very right of Figure 3: these variations make a systematic difference to how your visual system responds, enabling you to see them. If its spatial resolution were lower, your visual system would be sensitive only to variations further left in the figure.

Figure 3

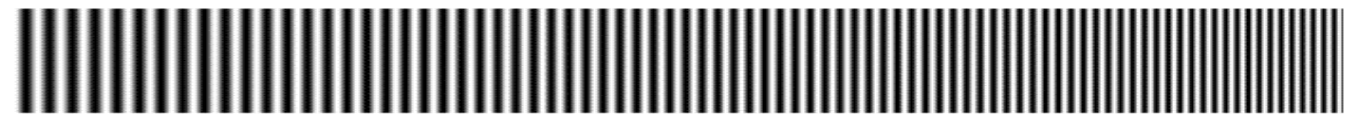

Because visual processes are insensitive to high rates of variation across space, they're insensitive to maximally determinate spatial properties. Consider an (artificially simple) example. You're presented with a line that's clearly visible and a short distance away. Call your visual response to the line's length ' $R$ ', and suppose that background conditions are such that $R$ occurs in the normal way. Conditional on $R$, there is a high probability that the line has any of a range of lengths, say between about 9.995 and $10.005 \mathrm{~cm}$. Conditional on $R$, the probability that the line has a length around the edge of this range is lower, and the probability that it has a length much outside the range is negligible. This is a corollary of the fact that, at the distance at which the line is presented, visual responses are not sensitive to variations which occur across less than about $0.01 \mathrm{~cm}$.

" Taking Sensitivity to be a condition on the visual presence of $P$ does not commit us to thinking that there is an analogous condition on propositional knowledge: if $p$ had not been the case, $S$ would not have believed that $p$. See Williamson 2002, Ch.7, for criticism of that idea. 
Call the line's maximally determinate length ' $L$ '. Whether or not the line has $L$ does not make a difference to whether $R$ occurs. There are possible worlds arbitrarily close to actuality, such that the object has a length which differs from $L$ by an arbitrarily small magnitude. At at least one of these close worlds, $R$ still occurs. So $R$ is not sensitive to $L$. By contrast, there is a determinable length to which $R$ is sensitive: the length between 9.995 and $10.005 \mathrm{~cm}$. There are worlds at which $R$ does not occur and the line is longer than $10.005 \mathrm{~cm}$ by an arbitrarily small magnitude. These worlds are closer to actuality than every world at which $R$ occurs in the normal way and the line is not between 9.995 and $10.005 \mathrm{~cm}$.

Strictly speaking this last claim needs qualifying. The probability distribution linking responses with lengths is continuous (typically it's Gaussian), so there is no sharp cut-off between probable and improbable lengths. To assess Sensitivity, we assess only worlds where conditions are such that if $R$ occurs, $R$ occurs in the normal way: many improbable worlds are excluded. Still, the notion of normalcy may be too vague to fix a definite point in the probability distribution beyond which improbable worlds are excluded. In that case, there will be no definite fact of the matter about the exact bounds of the determinable length to which $R$ is sensitive. Nonetheless, for any precisification of normalcy, there is a determinable length to which $R$ is sensitive.

Since visual spatial resolution is always limited, the argument generalises: for all visual responses, and all shapes and sizes, visual responses are sensitive to determinables not maximal determinates. This gives us reason to accept that Determinacy is violated by all normal cases of the visual presence of shape or size: in all such cases, the visually present properties are determinable, not maximally determinate. ${ }^{14}$

The foregoing is not decisive against Determinacy. To see this, consider a parallel with natural language. In some contexts though not in others, you can truly assert 'France is hexagonal' (Austin 1962). On one way of interpreting this utterance, in contexts where it's true it should be understood as an assertion that France is approximately hexagonal. By analogy, we might understand what is visually present not as $P$ simpliciter, but as approximately $P$. But on a different way of interpreting the utterance 'France is hexagonal', the semantic content asserted remains the same in different contexts; what changes is the degree of precision required for truth. On this approach, the assertion represents a property to which it's not sensitive: the property of being strictly-speaking hexagonal. The assertion may nonetheless be true, where truth need not be precise truth. By analogy, we might take visual experience to present things as they are as long as the property that's visually present approximates the property that's actually instantiated before you. So one could consistently

\footnotetext{
"Yablo (1992) argues that a determinable property $P$ (and not any determination of $P$ ) is causally efficacious of an effect $E$, where what makes a difference to $E$ is whether $P$ is instantiated, rather than whether any specific determination of $P$ is instantiated. If that's correct, determinable spatial properties rather than their maximal determinates are causally efficacious of visual effects, as well as reliably correlated with visual effects. But my argument does not turn on that.
} 
claim that visual experience normally presents things as they are, even though visual experiences are not sensitive to the spatial properties visually present in them. In particular one could insist on Determinacy, claiming that maximally determinate spatial properties are visually present in every visual experience that occurs under good conditions.

However, the analogy with natural language is limited. In the case of 'France is hexagonal', there is a plausible motivation for interpreting the utterance in a way which includes representation of a property to which it's not sensitive. The utterance shares the term 'hexagonal' with utterances which are sensitive to the property of being strictlyspeaking hexagonal, for instance competent utterances made in the practice of exact geometry. By contrast, no component of visual experience itself, or of its effects on belief and behaviour, is sensitive to a maximally determinate spatial property. The functional role of visual experience gives us no reason to introduce maximally determinate spatial properties into the lexicon of visual presence, so to speak. By contrast, the functional role of visual experience does give us reason to think that determinable spatial properties are visually present: visual experience is sensitive to determinable spatial properties.

It may be objected that the reasons for insisting on Determinacy lie in the introspectable character of visual experience, rather than its functional role. Here it's important to distinguish violations of Determinacy from other, related phenomena. It's sometimes said that the contents of consciousness are introspectably determinate; there is a definite fact about what the contents of a conscious episode are (Searle 1987). But if Determinacy is violated, this is not to say that it's vague or indeterminate which maximally determinate property is visually present; it's to say that no such property is present, and a determinable property is present. ${ }^{15}$

Ned Block objects to the idea that determinable properties figure in faithful descriptions of visual phenomenology, on the grounds that this is like describing visual experience in terms of disjunctive predicates:

By analogy, consider the supposition that something looks as follows: rectangular or triangular or circular. That disjunctive predicate does not describe one way that something can look—at least not in normal perceptual circumstances.

Block 2010: 52

Disjunctive predicates are inapt for describing the visual experience of a single shape or size. And it's true that, for any claim that the environment instantiates a determinable $D$, there is an extensionally equivalent disjunctive claim that the environment instantiates one or other

\footnotetext{
is Nor does the account I've given require that it's indeterminate which determinable property is visually present. As I said, given vagueness in the notion of normal background conditions, there may be no definite fact of the matter about which determinable a visual response is sensitive to. But for any precisification of normalcy, there is a definite determinable to which a response is sensitive. And we've been taking Sensitivity as at most a necessary condition on the visual presence of a property. Further conditions might select a definite determinable as the property which is visually present, even if Sensitivity does not.
} 
of $D^{\prime}$ s determinations. However, it's hard to see how this could undermine the mention of determinables in describing visual phenomenology. For any claim that the environment instantiates any property $P$, there is an extensionally equivalent disjunctive claim that the environment instantiates either $P$ or an impossible attribute. This suggests that it matters which of several extensionally equivalent descriptions of the environment we use, if we're to characterise visual experience faithfully. A determinable property is not merely a disjunctive property; determinable properties have a certain unity which merely disjunctive properties lack (§2). So to characterise experience in terms of a determinable is not to characterise it in terms of a disjunctive predicate. Rather, where a determinable shape is visually present, we must mention this single, unified shape in a faithful characterization of visual phenomenology; this requires no mention of the more specific disjoint possibilities consistent with something's having this shape.

If there is to be introspective support for Determinacy, this support will have to be more positive; it will have to consist in one's introspecting that certain maximally determinate properties are visually present. Here one might appeal to a claim of Timothy Williamson's:

In normally good circumstances I look at a sheet of paper with a black circle on a white background. To me, the black patch looks perfectly circular. It does not look imperfectly circular (almost but not quite perfectly circular). Nevertheless, ... [e]ven if by some miracle the patch is in fact perfectly circular, I cannot know by looking that it is perfectly circular.

Williamson 2013b: 85

The phrase 'looks perfectly circular' requires some care. Something may look perfectly circular, in that the way it looks is similar to the way something perfectly circular looks. (Compare: A drooping tree may look sad, in that the way it looks is similar to the way a sad person looks.) In that sense the black patch looks perfectly circular, as long as the black patch is visually indiscriminable from a patch which is in fact perfectly circular. But that is not the reading of 'looks perfectly circular' which Williamson requires. He cites his experience of the patch in defence of the following claim: there is a single exact property which the object of normal visual experience 'appears to have' (2013b: 85). His claim is that the patch appears to be perfectly circular.

I doubt that any standard use of 'appears to be ...' or 'looks to be ...' entails that a property is visually present. But visually present properties are some of the properties which things visually appear to have (§1), and perfectly circular is a maximally determinate shape. So one might take Williamson's claim as some support for Determinacy, the more general principle that wherever a determinable property $P$ is visually present in good viewing conditions, a determination of $P$ is visually present. However, we've already seen reason to doubt that introspection could provide this kind of positive support for 
Determinacy: even in good conditions like those of First ... Last, introspection does not put you in a position to tell exactly which properties are visually present $(\S 4)$.

The argument for this limit to introspection was as follows: if in good conditions introspection specified accurately exactly which properties are visually present to you, this would enable you to detect differences among the visually present properties of neighbouring stimuli in cases like First ... Last; by Transparency* you cannot do that. For example, suppose you're presented with a series of roughly circular patches, $C 1, C 2 \ldots C L$, under uniformly good viewing conditions. No neighbours in the series visibly differ in shape, but $C 1$ looks as circular as anything does, while $C L$ is visibly noncircular. The visually present shapes of some neighbouring patches must differ. By Transparency* you cannot detect these differences introspectively. So you cannot tell, introspectively, exactly which shapes are visually present. For instance you cannot tell whether the shape perfectly circular is visually present.

This argument is an inference to the best explanation: the best explanation of why you cannot detect differences among the visually present shapes of neighbouring patches is that introspection does not put you in a position to tell exactly which monadic shapes are visually present. One might object that a good explanation does not require ignorance about every visually present shape in the series: to explain why you cannot detect the difference between visually present shapes of neighbouring patches, we need only postulate ignorance about one member of each pair of different, neighbouring visually present shapes. For instance, suppose the visually present shapes of $C 1$ and $C 2$ differ. In principle you might introspect that the visually present shape of $C 1$ is perfectly circular, yet fail to introspect that the visually present shape of $C 2$ differs from this, because you cannot introspect exactly what the visually present shape of $C 2$ is. According to this line of objection, perfectly circular is an exceptional property, in that you can introspect that exactly this property is visually present, though you cannot introspect exactly which other properties are visually present, including the visually present shape of $C 2$.

Now perfectly circular is an exceptional property in that it's a canonical shape. But it is hard to see how a property's being canonical could be relevantly exceptional, such that you can introspect that exactly this property is visually present, whereas you cannot introspect that other, less canonical properties are visually present. In general canonical categories have the opposite effect, encouraging imprecision rather than added precision. We include things in the category for which the canonical property is paradigmatic despite their differing from the paradigm, as when we call printed shapes, which are only roughly circular, 'circular'. (This may be why we say that things 'look perfectly circular', and not that they 'look imperfectly circular', where 'look perfectly circular' is understood comparatively.) The canonical status of a property does sometimes help us to identify it precisely, where identifying the property is a matter of naming it. The phrase 'perfectly circular' gives us a 
precise way of identifying perfect circles which we lack for less regular shapes. For instance neither 'imperfectly circular' nor 'slightly elliptical' picks out a single exact shape. But it is implausible that this sort of linguistic phenomenon is responsible for our most fine-grained introspective capacities - on the contrary, it's a truism that we can detect myriad differentiations in our perceptual experiences which we lack the vocabulary to express.

It is hard to see, then, how one could sustain a claim to find introspective support for Determinacy, in face of the argument of $\S 4 .{ }^{16}$ Rather, introspection is neutral on the question whether maximally determinate properties are visually present. Nor does anything about the functional role of visual experience motivate the claim that maximally determinate properties are visually present. By contrast, the functional role of visual experience does provide a reason to think that determinable spatial properties are visually present: visual experiences are sensitive to determinable spatial properties. So a well motivated account of visual presence should reject Determinacy and appeal, as $\S 3$ did, to the presence of determinable properties.

If introspection is neutral on the question whether maximally determinate properties are visually present, why is it tempting to think we have introspective evidence for Determinacy? One source of this temptation may lie in the fact, noted above, that objects do look like objects with certain maximally determinate properties - for instance they look perfectly circular - though no such properties are visually present. I suspect another source of the temptation lies in the lingering role of impressions in our thinking. If we reify visual experience, it's easy to confuse determinacy in experience with the determinacy of its objects. It's natural to assume that each object you see does have some determinate shape. And when reflection on experience reveals such an object, it's tempting to take this object as an impression, an experiential item whose entire character is present to the mind, rather than as a physical object whose character is experienced only partially.

If the properties visually present to us are determinables to which our experiences are sensitive, as I've argued, then we have a further reason for rejecting Access that's independent of the challenge posed by cases like First ... Last. Where two things are very similar, their visually present properties will often be different but compatible: they will correspond to overlapping regions of property space. Where objects are very similar in length, say, it's highly probable that their visually present lengths will differ, and highly

\footnotetext{
"Thanks to Williamson for an objection along the lines just discussed. Williamson does not make a universal claim like Determinacy. He claims only to be able to tell that there are some cases in which normal visual appearances are maximally specific. My claim about the limits of introspection is also consistent with Williamson's (1990) proposal that experiential characters are 'subjective' in the sense that 'they are identical if and only if they are indiscriminable under all presentations'. I've argued that, in good viewing conditions, you cannot discriminate between some distinct experiential characters by identifying exactly which properties are visually present in them. This is consistent with Williamson's proposal that those characters must, if distinct, be discriminable under some other presentation. My claim about the limits of introspection is also consistent with, but not entailed by, Williamson's (2000) claim that experiences are not 'luminous': no experience is such that if you have it you can know you have it.
} 
improbable that their visually present lengths will be incompatible, in any given background condition within the normal range.

For instance, suppose $a$ is $10.0048 \mathrm{~cm}$ long and $b$ is $10.0052 \mathrm{~cm}$ long. Within the range of normal conditions, the visually present length of $a$ might be between 9.9949 and $10.0049 \mathrm{~cm}$, and the visually present length of $b$ might be between 10.0051 and $10.0151 \mathrm{~cm}$. But that's highly improbable. Conditions must be barely such that the experience of $a$ occurs in the normal way, since $a$ has a determinate length at the upper extreme of the lengths to which this experience is sensitive. And conditions must be barely such that the experience of $b$ occurs in the normal way, but the reason here is the opposite: $b$ has a length at the lower extreme of the lengths to which the experience of $b$ is sensitive. That conjunction of conditions is highly improbable. It's far more probable that the visually present lengths of $a$ and $b$ are different but compatible (Figure 4).

Figure 4

\begin{abstract}
Here the determinate length of each stimulus lies at a similar point within the range corresponding to the determinable length to which each response is sensitive. Since the stimuli are similar in length, the responses are sensitive to different but compatible lengths.
\end{abstract}

Length to which visual response is sensitive

Determinate stimulus length

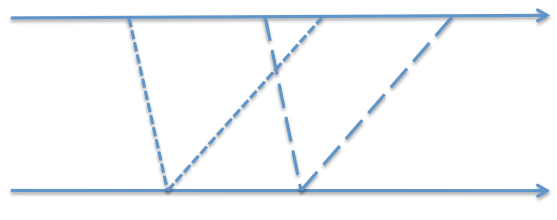

Stimulus 1 Stimulus 2

Now where objects are very similar in length, those objects do not visibly differ in length. So by Transparency*, the visually present lengths of such objects do not seem introspectively to differ. So we have a reason to deny Access that's independent of the challenge posed by series like First ... Last: if the properties visually present to us are properties to which our experiences are sensitive, then where two things are very similar, there is often an introspectively inaccessible difference between their visually present properties. The account I proposed in $\S \S 3-4$ is independently well motivated, both in its rejection of Determinacy and in its rejection of Access.

\footnotetext{
"Suppose you find yourself in this improbable situation, and $a$ and $b$ look to differ in length, as my account predicts. Still, you cannot discriminate the lengths of $a$ and $b$, in the sense of knowing that these lengths differ, for you fail standard conditions on knowledge. Had conditions been just a little different, and so abnormal, you might have had the same experiences while the lengths to which they're sensitive were not instantiated. So if $a$ and $b$ had not differed in length, you would have judged them distinct all the same: you fail Nozick's (1981) sensitivity condition on knowing. And if you judge $a$ and $b$ distinct in length, on the basis of your visual experience, you judge in a way which could easily have been false: you fail a safety condition on knowledge (Williamson 2002). Jeremy Goodman incorporates a margin of safety within unspecific appearances themselves, in order 'to vindicate ... the normality of our knowing that things are as they appear' (2013: 33). That strikes me as optional. Perhaps there are normal circumstances in which things are as they appear, but one cannot know that they are so. After all, knowledge is but one end among many for which visual appearances are useful, and other ends may not require analogues of safety.
} 


\section{Determinacy and colour experience}

Under good viewing conditions you're presented with three colour samples, $a, b$ and $c$, such that $a$ does not visibly differ in colour from $b$, nor $b$ from $c$, but $a$ visibly differs in colour from $c$. So the visually present colour of $a$ differs from that of $c$, and on pain of incoherence, the visually present colour of $b$ must differ from both. In principle, as I said, we could make sense of this situation in the same way as First ... Last: the visually present colours of $a$ and $c$ are incompatible; the visually present colour of $b$ differs from those of $a$ and $c$, but it is not incompatible with them; someone judging according to Discrimination can then discriminate $a$ from $c$ with respect to colour, but she cannot discriminate $b$ from either.

However, this solution requires that Determinacy is false with respect to colour experience. Now visual sensitivity to spectral properties of the light is limited, just as visual sensitivity to spatial properties is limited. This is in part because all visual processing is noisy. Noise is those collateral aspects of the visual signal from which the element of the signal produced by a stimulus must be distinguished, if the character of the stimulus is to be detected. This includes both external noise - collateral aspects of the light array - and internal noise - endogenously produced variation in a processing channel. As a result of noise, which colour experiences you have under normal conditions is determined, in part, by factors other than the spectral properties of the light reflected at a surface. Therefore, no colour experience is reliably correlated with maximally determinate spectral properties of the light reflected at a surface. One might argue on these grounds that visual responses are insensitive to maximally determinate colours, and that Determinacy is therefore false with respect to colour experience. (Cf. Hellie 2005: 489-492, for a related argument that colour experience is unspecific.) But given some fairly standard views about the nature of colour, insensitivity to spectral properties of the light does not entail insensitivity to things' colours.

There are of course many possible views about the nature of colour, but we can appreciate the issues here by considering just two broad types of view:

Disposition For a surface to have a certain colour is for that surface to be disposed to cause a certain experiential response in normal human observers under normal viewing conditions. ${ }^{18}$

According to Disposition, it's a necessary truth that some experiential response is sensitive to every colour, however determinate: for there to be a colour $C$ is, inter alia, for there to be a type of experience $E$ such that, in background conditions such that $E$ occurs in the normal way, $E$ would not have occurred if $C$ had not been instantiated. According to Disposition, the limited sensitivity of vision gives us no reason to reject Determinacy for the colours. ${ }^{19}$

\footnotetext{
"sor views in this spirit, see e.g. McGinn 1983, Smith 1990.

1" As well as arguing that colour experiences are not reliably correlated with maximally specific colours, Hellie appeals to the claim that noise is 'phenomenally manifest' in colour experience. $\mathrm{He}$ describes 'a subtle "flickering" or "crepitation", of the sort manifest in a snowy, poorly tuned rabbit-
} 
The case against Determinacy for the colours is better if we assume:

Reflectance For a surface to have a certain colour is for that surface to be disposed to reflect the light in some way..$^{20}$

Since visual experiences are insensitive to exact ways of reflecting the light, given Reflectance visual experiences are insensitive to exact colours. However, it's not obvious that visual experiences are therefore insensitive to maximally determinate colours, because it's not obvious how to understand the determination space for colours, if we endorse Reflectance. It's standard to assume that every colour is a determinate of the determinable coloured. Indeed, it's not clear in what sense a theory recognises a determinable-determinate structure for the colours, unless it's consistent with that assumption. On the face of it Reflectance does not meet this constraint.

Recall that a colour $C$ determines a colour $D$ if and only if $C$ differs in nature from $D$ only along the determination dimensions of hue, saturation and brightness, such that the values along these dimensions consistent with instantiating $C$ are a proper subset of the values consistent with instantiating $D$. Now there are differences between exact ways of reflecting the light which have no principled connection with any of the differences in virtue of which we experience different values for hue, saturation, or brightness. Light distributions with various different constituent wavelengths form metamers - that is, these light distributions are indistinguishable in colour to a human observer. In particular, there are infinitely many different triples of spectral lights which produce metamers when the three lights are combined (Hardin 1993: 28). (Triples, because of the trichromatic structure of human vision. The constraint is just that one of the three lights must be mostly blue, one mostly green, and one mostly red.) On the face of it, there could be no principled reason to represent the differences between many such triples by any particular transformation along the dimensions of hue, saturation and brightness. The determination dimensions of colour have the wrong structure to capture this difference in the exact properties of the light. So if with Reflectance - we take every way of reflecting the light to fix a colour, there are colours which are not determinations of the determinable coloured.

Suppose you endorse Reflectance and you also want to be able to talk in terms of a determination space for the colours - for instance, you want to argue against Determinacy for the colours. Then you have two clear options at this point. First, you could propose a

ears TV'. This, he claims, occludes the colours before us, so that normal visual experience fails to specify exactly which colours they are (2005: 493). Let's accept, for the sake of argument, that there is flickering of this kind in colour experience. Whether it makes sense to suppose that this occludes maximally determinate colours depends on our broader views about the nature of colour. For instance, Disposition is inconsistent with this proposal. According to Disposition, to have a maximally determinate colour is to cause experiences of a certain kind under normal conditions. So any flickering or crepitation in normal colour experience fixes the nature of the colours, rather than occluding them.

${ }^{20}$ For views in this spirit, see e.g. Tye 2000, Byrne and Hilbert 2003. 
revision of our naïve view about determination dimensions of colour. You could argue that the real determination dimensions of colour are not hue, saturation and brightness, or anything of their ilk. Rather, the determination dimensions of colour are to be given in terms of response-independent, physical properties of the light. Presumably there will then be determinate colours to which human visual experience is altogether insensitive." Secondly, you could retain the naïve determination dimensions for colour, and adopt a qualified version of Reflectance:

Reflectance* $^{*}$ For a surface to have a certain colour is for that surface to be disposed to reflect the light in a way $W$, where to reflect the light in way $W$ is to reflect it in any of a set of ways the differences between which have no principled connection with differences in hue, saturation or brightness.

According to Reflectance*, many different ways of reflecting the light fix just one colour, but the colours cut finer than metamers. The colours cut finer than metamers, because there are some invisible differences between ways of reflecting the light which do have a principled connection with differences in hue, saturation or brightness.

There are, then, possible views about the nature of colour according to which insensitivity to the light entails insensitivity to maximally determinate colours. If we endorse one of these views, we can appeal to a sensitivity condition on visual presence to motivate rejecting both Determinacy and Access for the colours, and thereby to defend the idea that colours are visually present. But these are quite specific views about the nature of colour. It should be accordingly controversial whether there is a good case against Determinacy and Access for the colours. The trouble here lies in the fact that, on some views, either the colours themselves or their determination space cut no finer than normal human responses.

I won't defend any particular view about the nature of colour here. Rather, I'd like to note a more abstract point about the visual presence of a property. It's traditional to suppose that what is visually present is laid bare before us in all its detail. So we might naturally think that colours are more apt than spatial properties for visual presence, because the nature of colour seems so intimately tied to visual experience. In fact, on the contrary, an intimate connection between the nature of colour and visual experience would make it harder to defend the idea that colours are visually present to us. For we can achieve a well motivated account of visual presence where we can reject Determinacy. On this account, experience provides a merely partial insight into the property which is present, leaving its

\footnotetext{
"This position is in the spirit of Kalderon 2007. He argues that every experienced colour is a determinable of some physical determinate, and he claims that the structure of traditional colourspace is response-dependent, while the colours themselves are not. Yablo (1995) likewise argues that colours are 'nonphysical determinables' of 'microphysical determinates'. Yablo does not worry about the determination dimensions of colour, but this is because his notion of 'determination' doesn't distinguish determinables from merely disjunctive properties (see $§ 2$ above).
} 
determinations obscure to us. As a consequence, visual presence puts us in a position to form only inexact true beliefs both about our environment and about our experiences. ${ }^{2}$

\section{REFERENCES}

Austin, J. 1962. How To Do Things With Words. Oxford: Clarendon Press. Block, N. 2010. Attention and mental paint. Philosophical Issues 20: 23-63. Byrne, A. \& Hilbert, D. 2003. Color realism and color science. Behavioral and Brain Sciences 26, 3-64. Campbell, J. 2002. Reference and Consciousness. Oxford: Clarendon Press.

DeValois, R. \& DeValois, K. 1988. Spatial Vision. Oxford: OUP. Dretske, F. 1995. Naturalizing the Mind. Cambridge MA: MIT Press.

Fodor, J. 1990. A Theory of Content and Other Essays. Cambridge MA: MIT Press.

Funkhouser, E. 2006. The determinable-determinate relation. Nous 40 (3): 548-569.

Goodman. J. 2013. Inexact knowledge without improbable knowing. Inquiry 56:1, 30-53.

Goodman, N. 1966. The Structure of Appearance (2w edition). Indianapolis: Bobbs-Merrill Co.

Graff, D. 2001. Phenomenal continua and the sorites. Mind 110 (440): 905-935

Hardin, C. 1993. Color for Philosophers: Unweaving the Rainbow. Indianapolis: Hackett.

Hellie, B. 2005. Noise and perceptual indiscriminability. Mind 114 (455): 481-508.

Hume, D. 1740/1978. A Treatise of Human Nature, ed. R. Selby-Bigge / P. Nidditch. Oxford: OUP.

Jackson, F. \& Pinkerton, R. 1973. On an argument against sensory items. Mind 82: 269-272.

Johnson, W. 1921. Logic (Vol. 1). Cambridge: Cambridge University Press.

Kalderon, M. 2007. Color primitivism. Philosophical Review 116 (4): 563-601.

Lewis, D. 1973. Counterfactuals. Oxford: Basil Blackwell.

Mills, E. 2002. Fallibility and the Phenomenal Sorites. Nous 36: 384-407.

Moore, G. 1903. The refutation of idealism. Mind, New Series, 12 (48): 433-453.

Nozick, R. 1981. Philosophical Explanations. Cambridge MA: Belknap Press.

Peacocke, C. 1983. Sense and Content. Oxford: OUP.

Peacocke, C. 1989. Perceptual content. In J. Almog, J. Perry \& H. Wettstein (eds.), Themes from

Kaplan. Oxford: OUP.

Prior, A. 1949. Determinables, determinates and determinants (Part I). Mind 58(229): 1-20.

Searle, J. 1987. Indeterminacy, empiricism, and the first person. Journal of Philosophy, 84(3):123-146. Siegel, S. 2006. Which properties are represented in perception? In T.S. Gendler and J. Hawthorne (eds.) Perceptual Experience. Oxford: Clarendon Press.

Siegel, S. 2010. The Contents of Visual Experience. Oxford: OUP.

Smith, A.D. 1990. Of primary and secondary qualities. Philosophical Review 99 (2): 221-254.

Stazicker, J. 2011. Attention $\mathcal{E}$ the Indeterminacy of Visual Experience. UC Berkeley PhD dissertation.

Strawson, P. 1979. Perception and its objects. In Perception and Identity: Essays presented to A.J. Ayer.

London: MacMillan Press.

Stroud, B. 2009. Scepticism and the senses. European Journal of Philosophy, 17(4): 559-570.

Travis, C. 2004. The silence of the senses. Mind 113 (449): 58-94.

Williamson, T. 2002. Knowledge and its Limits. Oxford: OUP.

Williamson, T. 2013a. Gettier cases in epistemic logic. Inquiry 56:1, 1-14.

Williamson, T. 2013b. Response to Cohen, Comesaña, Goodman, Nagel, and Weatherson on Gettier cases in epistemic logic. Inquiry 56:1, 77-96.

Wright, C. 1975. On the coherence of vague predicates. Synthese 30: 325-365.

Yablo, S. 1992. Mental causation. Philosophical Review 101(2). 245-280.

Yablo, S. 1995. Singling out properties. Philosophical Perspectives 9: 477-502.

\footnotetext{
2 For helpful questions, thanks to participants at several events: in 2010, the EXRE / University of Fribourg workshop on Phenomenal Presence; in 2013, a Mind Network meeting in London, a Work in Progress Seminar at the University of Reading, and the Barnard-Columbia Perception Workshop. Thanks to the University of Fribourg and Fabian Dorsch for their hospitality, and to the UC Berkeley Graduate Division for sponsorship. For other helpful comments and discussions, thanks to Ned Block, Michael Caie, John Campbell, Tim Crane, Craig French, Jeremy Goodman, Benj Hellie, Mark Eli Kalderon, Tamar Lando, Geoffrey Lee, John Morrison, Martin Nida-Rümelin, Alva Noë, Barry Stroud and Timothy Williamson. I'm especially grateful to MGF Martin for encouraging me to tackle some of the issues discussed here, and to the editors of this volume for their patience.
} 